\title{
Enhancing the reporting and transparency of rheumatology research: a guide to reporting guidelines
}

\author{
Robin Christensen ${ }^{1,2 *}$, Henning Bliddal' and Marius Henriksen'
}

\begin{abstract}
Manuscripts and abstracts from biomedical journals frequently do not contain proper information for meeting required standards and serving the multiple needs of their end users. Reporting guidelines and checklists help researchers to meet those standards by providing rules or principles for specific research areas. Rheumatology research includes a broad range of heterogeneous research areas, each with its own requirements, producing several distinct categories of articles. Our objectives with this article are to raise awareness of the existence and importance of reporting guidelines, to present a structured overview of reporting guidelines that rheumatology journals could apply, and to encourage their use by journal authors, editors, and reviewers, including those of Arthritis Research \& Therapy. Internationally recognized reporting guidelines exist for a diversity of research areas. We encourage colleagues to consult the 'Enhancing the QUAlity and Transparency Of health Research' (EQUATOR) network when writing scientific papers. EQUATOR is an international initiative that seeks to improve the reliability and value of biomedical research literature by promoting transparent and accurate reporting of studies. We propose specific reporting guidelines for a number of study designs: animal research, randomized trials, reliability and agreement studies, systematic reviews with and without meta-analyses, diagnostic test accuracy studies, and also observational research including cross-sectional, cohort, and case-control studies. We encourage authors, editors, and reviewers to adhere to and enforce the use of the appropriate guidelines when writing, reading, and reviewing scientific papers.
\end{abstract}

\section{Introduction}

Quite often, research reports and abstracts do not contain proper information for meeting required standards and serving the multiple needs of their end users [1], be they researchers or health care providers. Poor methodology and reporting are widespread [2]. As a result, published studies often cannot be replicated by researchers, translated into clinical practice, or used to inform public health policy. Guidelines and checklists help researchers meet certain standards by providing sets of rules or principles for implementing best practices in a particular area [3].

Most trialists and many research managers know the advantages associated with using the CONSORT (CONsolidated Standards Of Reporting Trials) statement when writing a scientific paper. CONSORT is a statement

${ }^{*}$ Correspondence: Robin.Christensen@Frh.Regionh.DK

'The Parker Institute, Department of Rheumatology, Copenhagen University

Hospital, Frederiksberg, Copenhagen F, Denmark

Full list of author information is available at the end of the article intended to assess the validity of results from randomized controlled trials (RCTs) and to improve their reporting, enabling readers to understand a trial's design, conduct, analysis, and interpretations [4]. However, many do not know about the umbrella organization that CONSORT is part of, the 'Enhancing the QUAlity and Transparency Of health Research' (EQUATOR) network [5]. The EQUATOR network is an international initiative that seeks to improve the reliability and value of biomedical research literature by publishing a variety of different reporting guidelines that promote the transparent and accurate reporting of research studies [5].

The objectives of this commentary are to raise awareness of the existence and importance of reporting guidelines within the various areas of research and to present them in a structured overview so that journal authors, editors, and reviewers - including those of Arthritis Research $\mathcal{E}$ Therapy - might more easily apply them.

\section{Applying reporting guidelines}

Reporting guidelines are statements that provide advice on how to report methods and findings of research [5]. 
Usually in the form of a checklist, flow diagram or explicit text examples, they specify a minimum set of items required for a clear and transparent account of what was done and what was found in a study. Rheumatology and related research includes a broad range of heterogeneous research areas, each generating a category of articles with separate requirements. All reporting categories of articles should conform to the 'Uniform Requirements for Manuscripts Submitted to Biomedical Journals', developed by the International Committee of Medical Journal Editors [6]. Nevertheless, guidelines can still be specifically tailored to different study types while conforming to these requirements, providing more focused guidance to writers and researchers - information that is valuable even at the protocol stage [7]. It is not uncommon that scientists set up a research project in a manner that will inevitably lead to unsatisfactory reporting; that is, either the data are not there or are there in the wrong way.

The EQUATOR network library currently provides reporting guidelines for a number of study types, such as 'experimental studies', 'observational studies', 'diagnostic accuracy studies,' 'biospecimen reporting,' 'reliability and agreement studies', 'systematic reviews', 'qualitative research', 'mixed methods studies,' 'economic evaluations', and 'quality improvement studies' [8].

\section{Recommended statements}

Table 1 presents a structured overview of research designs that could appear in a journal like Arthritis Research $\mathcal{E}$ Therapy. The guidelines illustrated in Table 1 are already widely endorsed by other journals; thus, authors who submit papers to the EQUATOR-endorsing journals would be expected to follow the pertinent reporting statement. The goals associated with the EQUATOR initiative are many, but the overarching goal at least, as we see it - is to prevent authors from omitting crucial information in the description of research methods and interventions, in order to avoid disappointing results in primary or secondary (post hoc) analyses. Therefore, we recommend that all reporting researchers search the EQUATOR network for its many helpful guidelines for different study types.

Table 1 reflects what we currently see as the most important statements that could apply for a journal like Arthritis Research \& Therapy. The CONSORT statement will probably be perceived as the most obvious [4], and we are confident that most of our publishing research colleagues are aware of this statement already. We hope they agree that the use of the CONSORT statement checklist is helpful even for purposes such as structuring an article outline and for supervising junior colleagues and students preparing their first randomized controlled trial paper. The STROBE (Strengthening the Reporting of Observational Studies in Epidemiology) statement describes what is important to report when writing an epidemiological paper [9]. The STROBE statement consists of a checklist of 22 items relating to the title, abstract, introduction, methods, results, and discussion sections of articles [9]. Of those 22 items, 18 are common to cohort studies, case-control studies, and crosssectional studies, and 4 are specific to each of the 3 study designs. The STROBE statement intends to improve the reporting of all three study designs, thereby facilitating critical appraisal and interpretation of findings by reviewers, journal editors, and readers. The PRISMA (Preferred Reporting Items for Systematic reviews and Meta-Analyses) statement is an evolution of the original QUOROM (Quality of Reporting of Meta-analyses) guideline on how to report systematic reviews and metaanalyses [10]. The PRISMA statement focuses mainly on meta-analysis of randomized controlled trials but is also relevant for meta-analyses of observational studies; the PRISMA statement does not replace the MOOSE (Metaanalysis Of Observational Studies in Epidemiology) statement [11]. The PRISMA statement consists of a 27-item checklist and a 4-phase flow diagram as a guide to authors.

Guidelines can also improve diagnostic test accuracy studies, which are often submitted to medical journals. The STARD (Standards for Reporting of Diagnostic Accuracy) statement aims to improve the quality of reporting of studies concerning diagnostic accuracy [12]. STARD introduces a checklist of 25 items and suggests a flow diagram that authors can use to ensure that all relevant information is present. STARD contains a clarification of the meaning, rationale, and optimal use of each item on the checklist, as well as a short summary of the available evidence on bias and applicability [12].

An essential requirement of all outcome measures in biomedical research is that they be valid and reproducible. Overall reproducibility concerns the degree to which repeated measurements provide the same results. Reproducibility covers both agreement and reliability concepts that are not easily distinguished from each other. Reliability may be defined as the ratio of variability between subjects (for example, patients) or objects (for example, ultrasound assessments) to the total variability of all measurements in the sample; reliability can be defined as the ability of a measurement to differentiate between subjects or objects [13]. On the other hand, agreement is the degree to which scores or ratings are identical. Both concepts are important, because they provide information about the quality of measurements. Reliability is highly dependent on the heterogeneity of the study sample, while the agreement, based on measurement error, is more a pure characteristic of the measurement instrument [14]. Partly overlapping the STARD statement, GRRAS (Guidelines for Reporting 
Table 1. Proposed subtitling and guidelines for various research project categories

\begin{tabular}{|c|c|c|c|c|c|c|}
\hline $\begin{array}{l}\text { Research } \\
\text { project }\end{array}$ & $\begin{array}{l}\text { Randomized } \\
\text { trials }\end{array}$ & $\begin{array}{l}\text { Observational } \\
\text { studies }\end{array}$ & $\begin{array}{l}\text { Systematic reviews } \\
\text { and meta-analyses }\end{array}$ & $\begin{array}{l}\text { Diagnostic } \\
\text { test studies }\end{array}$ & $\begin{array}{l}\text { Reliability and } \\
\text { agreement studies }\end{array}$ & $\begin{array}{l}\text { Research in } \\
\text { animal studies }\end{array}$ \\
\hline $\begin{array}{l}\text { Last part of } \\
\text { suggested title (that } \\
\text { is, Title Suffix) }\end{array}$ & $\begin{array}{l}\text { A randomized } \\
\text { controlled trial }\end{array}$ & $\begin{array}{l}\text { A cross-sectional study } \\
\text { A cohort study } \\
\text { A case-control study }\end{array}$ & $\begin{array}{l}\text { A systematic review } \\
\text { and meta-analysis } \\
\text { of randomized trials }\end{array}$ & $\begin{array}{l}\text { A diagnostic } \\
\text { accuracy study }\end{array}$ & $\begin{array}{l}\text { A reliability and } \\
\text { agreement study }\end{array}$ & $\begin{array}{l}\text { An in vivo animal } \\
\text { research study }\end{array}$ \\
\hline $\begin{array}{l}\text { Statement or } \\
\text { guideline to consult }\end{array}$ & $\begin{array}{l}\text { CONSORT } \\
\text { statement }\end{array}$ & STROBE statement & PRISMA statement & STARD statement & GRRAS statement & ARRIVE statement \\
\hline PubMed ID & PMID: 20332511 & PMID: 17938389 & PMID: 19622512 & PMID: 12513067 & PMID: 21130355 & PMID: 20613859 \\
\hline
\end{tabular}

ARRIVE, Animals in Research Reporting In Vivo Experiments; CONSORT, CONsolidated Standards Of Reporting Trials; GRRAS, Guidelines for Reporting Reliability and Agreement Studies; PRISMA, Preferred Reporting Items for Systematic reviews and Meta-Analyses; STARD, Standards for Reporting of Diagnostic Accuracy; STROBE, Strengthening the Reporting of Observational Studies in Epidemiology.

Reliability and Agreement Studies) includes a checklist with 15 issues that should be addressed when reliability and agreement are reported [13].

Finally, the ARRIVE (Animals in Research: Reporting In Vivo Experiments) guidelines [15] address previous inadequacies of biomedical journals that provided little or no guidance on what information to report when describing animal research. The ARRIVE guidelines consist of a checklist of 20 items describing the minimum information that all scientific publications reporting research using animals should include [15].

We anticipate some researchers would be looking for a general statement on 'Bench research' (that is, research exclusively conducted in laboratory settings), which unfortunately is not available yet - perhaps due to the multitude of different research methods that are difficult to standardize across disciplines. However, the REporting recommendations for tumour MARKer prognostic studies (REMARK) would be recommended in oncology [16] - principles that may also be applicable in rheumatology. Research in human biospecimens should follow the recommendations from BRISQ (Biospecimen Reporting for Improved Study Quality), which aims at improving the quality of research that uses human tissues subjected to collection, processing, and storage; it is crucial that information on the handling of biospecimens be reported in a thorough, accurate, and standardized manner [17].

\section{Discussion}

The aim of medical research is to advance scientific knowledge and thereby lead to more rational decision making and improvements in treating and preventing disease [2]. Despite the effort of researchers, editors, and peer reviewers, the quality of health-research reporting in journal articles has room for improvement [5]. We present six statements and guidelines that rheumatology journals like Arthritis Research \& Therapy would benefit from endorsing in the future. These six statements and guidelines, presented in Table 1, cover broad areas, such as how to report findings from randomized trials, observational studies, systematic reviews and meta-analyses, diagnostic tests, reliability and agreement studies, and animal studies. The statements specify a minimum set of items for reporting and can improve the accuracy and transparency of publications, thus facilitating easier and more reliable appraisal of quality and relevance [5].

Different researchers have different opinions about the role of publication guidelines [18]. In our experience, which is shared by many others, adherence to guidelines, even at the protocol stage, adds important details to the creative process, as it helps researchers consider what they will write in their final paper of the research project they propose, for instance in the 'Methods section' [7]. Following CONSORT, many other guidelines were developed; currently, there are probably 100 available for reporting different types of health research. Reporting guidelines may be regarded as a help for authors, editors and reviewers, and in particular, less-experienced younger colleagues.

A conventional view sees guidelines as a means of helping researchers to publish results as clearly as possible, in a way that facilitates interpretation and comparison with results from similar studies, and most guidelines are easy to employ [18]. Whatever the view on guidelines, clear reporting eases replication of studies, which is a fundamental principle in developing and applying scientific knowledge. Professor Doug Altman published in 2002 a thought-provoking article in JAMA titled 'PoorQuality Medical Research - What Can Journals Do' [2], in which he encouraged researchers and journal editors to remember that a study should not mislead; otherwise it could adversely affect clinical practice and future research [2].

We believe that the ability to self-correct is a prudent feature of science. Self-correction is often impeded by destruction of evidence, production of faulty evidence, and/or distortion of evidence [19]. Proper and accurate reporting of scientific data, results, and interpretations is key to ensuring that these impediments are addressed. 
Therefore, we encourage research colleagues (whether acting as authors or reviewers) and journal editors to endorse the use of guidelines and checklists as a critical filter when planning, performing, presenting, and publishing research data. One way of self-correction is to consider how the results should be reported properly and accurately as early as in the conception of a research project [7]. By integrating the recommendations early in the life cycle of the study, many methodological mistakes can be avoided that inevitably lead to unsatisfactory reporting, and will ultimately result in higher value of the study. We strongly believe that using these mechanisms from project conception to publication creates win-win situations for all end users: researchers, clinicians, and patients.

\section{Conclusion}

The abundance of available biomedical scientific literature and the increasing number of published articles - in rheumatology as in other fields - create a need for consistency in reporting in order to enhance evidence synthesis, ease clinical decision making, and inform healthcare policy makers. Internationally recognized reporting guidelines exist for a diversity of research areas, and we encourage authors, editors, and reviewers to adhere to and enforce the use of the appropriate guidelines when writing, reading, and reviewing scientific papers.

\section{Abbreviations}

ARRIVE, Animals in Research Reporting In Vivo Experiments; CONSORT, CONsolidated Standards Of Reporting Trials; EQUATOR, Enhancing the QUAlity and Transparency Of health Research; PRISMA, Preferred Reporting Items for Systematic reviews and Meta-Analyses; STARD, Standards for Reporting of Diagnostic Accuracy; STROBE, Strengthening the Reporting of Observational Studies in Epidemiology.

\section{Competing interests}

$\mathrm{RC}$ reports being involved in health-care initiatives and research that benefit from wide uptake of reporting guidelines (including Cochrane Collaboration, and the GRADE Working Group). HB reports that he has no competing interests in regard to this manuscript. MH reports that he has no competing interests in regard to this manuscript.

\section{Authors' contributions}

$\mathrm{RC}$ prepared the first draft of the manuscript and the other coauthors contributed to revised drafts and have approved the final version.

\section{Acknowledgements}

The Parker Institute is supported by grants from the Oak Foundation.

\section{Author details}

'The Parker Institute, Department of Rheumatology, Copenhagen University Hospital, Frederiksberg, Copenhagen F, Denmark. Institute of Sports Science and Clinical Biomechanics, University of Southern Denmark, Odense, Denmark.
Published: 28 February 2013

\section{References}

1. Chan AW, Altman DG: Epidemiology and reporting of randomised trials published in PubMed journals. Lancet 2005, 365:1159-1162.

2. Altman DG: Poor-quality medical research: what can journals do? JAMA 2002, 287:2765-2767.

3. Simera I, Moher D, Hoey J, Schulz KF, Altman DG: A catalogue of reporting guidelines for health research. Eur J Clin Invest 2010, 40:35-53.

4. Moher D, Hopewell S, Schulz KF, Montori V, Gotzsche PC, Devereaux PJ, Elbourne D, Egger M, Altman DG: CONSORT 2010 explanation and elaboration: updated guidelines for reporting parallel group randomised trials. BMJ 2010, 340:c869.

5. Altman DG, Simera I, Hoey J, Moher D, Schulz K: EQUATOR: reporting guidelines for health research. Lancet 2008, 371:1149-1150.

6. International Committee of Medical Journal Editors [http://www.icmje.org/]

7. Christensen $\mathrm{R}$, Langberg $\mathrm{H}$ : Statistical principles for prospective study protocols: design, analysis, and reporting. Int J Sports Phys Ther 2012, 7:504-511.

8. EQUATOR Network [http://www.equator-network.org/]

9. Vandenbroucke JP, von EE, Altman DG, Gotzsche PC, Mulrow CD, Pocock SJ, Poole C, Schlesselman JJ, Egger M: Strengthening the Reporting of Observational Studies in Epidemiology (STROBE): explanation and elaboration. Ann Intern Med 2007, 147:W163-W194.

10. Liberati A, Altman DG, Tetzlaff J, Mulrow C, Gotzsche PC, loannidis JP, Clarke M, Devereaux PJ, Kleijnen J, Moher D: The PRISMA statement for reporting systematic reviews and meta-analyses of studies that evaluate health care interventions: explanation and elaboration. Ann Intern Med 2009, 151:W65-W94.

11. Stroup DF, Berlin JA, Morton SC, Olkin I, Williamson GD, Rennie D, Moher D, Becker BJ, Sipe TA, Thacker SB: Meta-analysis of observational studies in epidemiology: a proposal for reporting. Meta-analysis Of Observational Studies in Epidemiology (MOOSE) group. JAMA 2000, 283:2008-2012.

12. Bossuyt PM, Reitsma JB, Bruns DE, Gatsonis CA, Glasziou PP, Irwig LM, Moher D, Rennie D, de Vet HC, Lijmer JG: The STARD statement for reporting studies of diagnostic accuracy: explanation and elaboration. Ann Intern Med 2003, 138:W1-12.

13. Kottner J, Audige L, Brorson S, Donner A, Gajewski BJ, Hrobjartsson A, Roberts C, Shoukri M, Streiner DL: Guidelines for Reporting Reliability and Agreement Studies (GRRAS) were proposed. J Clin Epidemiol 2011, 64:96-106.

14. de Vet $H C$, Terwee $C B$, Knol DL, Bouter $L M$ : When to use agreement versus reliability measures. J Clin Epidemiol 2006, 59:1033-1039.

15. Kilkenny C, Browne WJ, Cuthill IC, Emerson M, Altman DG: Improving bioscience research reporting: the ARRIVE guidelines for reporting animal research. PLOS Biol 2010, 8:e1000412.

16. McShane LM, Altman DG, Sauerbrei W, Taube SE, Gion M, Clark GM: Reporting recommendations for tumor marker prognostic studies (REMARK). J Natl Cancer Inst 2005, 97:1180-1184.

17. Moore HM, Kelly AB, Jewell SD, MCShane LM, Clark DP, Greenspan R, Hayes DF, Hainaut P, Kim P, Mansfield EA, Potapova O, Riegman P, Rubinstein Y, Seijo E, Somiari S, Watson P, Weier HU, Zhu C, Vaught J: Biospecimen reporting for improved study quality (BRISQ). Cancer Cytopathol 2011, 119:92-101.

18. Vandenbroucke JP: STREGA, STROBE, STARD, SQUIRE, MOOSE, PRISMA, GNOSIS, TREND, ORION, COREQ, QUOROM, REMARK... and CONSORT: for whom does the guideline toll? I Clin Epidemiol 2009, 62:594-596.

19. Ioannidis JP: Why most published research findings are false. PLoS Med 2005, 2:e124.

doi:10.1186/ar4145

Cite this article as: Christensen $\mathrm{R}$, et al.: Enhancing the reporting and transparency of rheumatology research: a guide to reporting guidelines. Arthritis Research \& Therapy 2013, 15:109. 